\title{
Aging kinetics of 14H-LPSO precipitates in Mg-Zn-Y alloy
}

\author{
*Di-qing Wan, Hou-bin Wang, Zhu-min Li, Ying-lin Hu, Jia-jun Hu, Yan-dan Xue, Lin-sen Wang, Kang-jin Fang \\ School of Materials Science and Engineering, East China Jiaotong University, Nanchang 330013, China
}

\begin{abstract}
Alloys with long-period stacking ordered structures (LPSO) have good properties and are highly regarded. Mg-Zn-Y alloy containing LPSO phase was prepared by the traditional casting method, and the aging heat treatment was performed at different temperatures and times. The microstructure and phase constitutions of the alloy were observed by means of optical microscopy and scanning electron microscopy methods. Results show the microstructure of as-cast Mg95.5Zn1.5Y3 mainly consists of $\alpha-\mathrm{Mg}$, W phases and LPSO phases. During the aging treatment, fine lamellar-shaped 14H-LPSO phase is formed at the grain boundaries and precipitates from the supersaturated magnesium matrix, and the volume fraction increases as the aging time increases. By controlling the aging time, $\mathrm{Mg}-\mathrm{Zn}-\mathrm{Y}$ alloys with different volume fractions of $14 \mathrm{H}$-LPSO phase were prepared. The aging kinetics equation of the $14 \mathrm{H}$-LPSO phase is summarized, that is $f=1-\exp \left(-0.2705 t^{0.6368}\right)$. The phase transformation mechanism of $14 \mathrm{H}-\mathrm{LPSO}$ in Mg95.5Zn1.5Y3 alloy can be described as the change of dislocation energy.
\end{abstract}

Key words: magnesium alloy; LPSO phase; aging kinetics equation

CLC numbers: TG146.22 Document code: A Article ID: 1672-6421(2020)01-042-06

$\mathrm{M}$ agnesium alloys have advantages of low density, high specific strength and specific stiffness, good damping, castability, machinability and easy recovery, and are regarded as engineering materials with the greatest potential after aluminum alloys. However, the lower hardness, modulus of elasticity, wear resistance, and high coefficient of thermal expansion of magnesium alloys limit their further application ${ }^{[1-4]}$. Mg-Zn-Re alloy has attracted more and more attention due to its special structural phase (long-period stacking ordered structures phase, LPSO) and good mechanical properties. A study found that when the volume fraction of LPSO phase in the magnesium matrix reaches $85 \%-90 \%$, the yield strength of extruded Mg89Zn4Y7 alloy can reach 480 $\mathrm{MPa}^{[5]}$. Onorbe E. ${ }^{[6]}$ prepared Mg100-3xY2xZn $x$ alloys (where $x=0.5,1$ and 1.5at.\%) with different LPSO phase contents by the in-situ method, and studied the effects of LPSO phase content on the microstructure and mechanical properties. The results indicate that with the increase of LPSO phase, the room temperature yield strength improved noticeably. LPSO can effectively

\section{*Di-qing Wan}

Male, Ph.D., Professor. Research interests: high strength and high corrosion resistant magnesium-based damping materials. His academic research has led to the publication of more than 60 technical papers.

E-mail: divadwan@tom.com

Received: 2019-09-24; Accepted: 2019-12-11 hinder the movement of dislocations, and at the same time can pin the grain boundaries, which is the root cause for improving the mechanical properties of materials ${ }^{[7]}$. Itoi ${ }^{[8]}$ first discovered 14H-LPSO phase in Mg97Zn1Y2 (at.\%) after suitable heat treatment. Xu C et al. ${ }^{[9]}$ studied the heat treatment of extruded $\mathrm{Mg}-\mathrm{Zn}-\mathrm{Y}$ alloy, and found the properties were significantly improved due to the distribution of fine LPSO phase at the grain boundary. The formation mechanism of 14H-LPSO phase suggests transformation from 18R-LPSO phase and precipitates in super-saturated $\alpha-\mathrm{Mg}$ solid solution through a modulated structure-like mechanism ${ }^{[10-12]}$. Generally, 14H-LPSO phase precipitated in the supersaturated $\alpha-\mathrm{Mg}$ solid solution requires two processes: nucleation and growing process. In recent studies, it was found that $14 \mathrm{H}$-LPSO phase grew rapidly in the $\alpha-\mathrm{Mg}$ matrix ${ }^{[13-14]}$. However, the theoretical study on nucleation of $14 \mathrm{H}-\mathrm{LPSO}$ phase during precipitation is still unclear.

Based on this background, in this study, the precipitation process of $14 \mathrm{H}-\mathrm{LPSO}$ phase during aging treatment, and the aging kinetics equations about $14 \mathrm{H}$-LPSO phase were studied. The theoretical study about nucleation of 14H-LPSO phase during precipitation was analyzed. At the same time, the relationship between the concentration of 14H-LPSO phase in the super-saturated $\alpha-\mathrm{Mg}$ solid solution and $\mathrm{Mg}$ alloys was discussed. 


\section{Experiment}

Mg95.5Zn1.5Y3 (in at.\%) alloys were prepared by conventional casting methods using purity Mg (99.95wt.\%), Zn (99.9wt.\%) and $\mathrm{Mg}-25 \% \mathrm{Y}$ (wt.\%) master alloys as raw materials. The melting process is performed in a SG-3-10 well-type electrical resistance furnace with argon protective gas. Firstly, the pure magnesium and pure zinc were put in a crucible pre-heated at $300{ }^{\circ} \mathrm{C}$. Then, the temperature was increased to $720{ }^{\circ} \mathrm{C}$ to ensure that the alloy was completely melted. Then, the $\mathrm{Mg}-$ $25 \%$ Y master alloy was added and held for 10-15 min. Finally, the molten metal was poured into an iron mold. The casting size is $220 \times 29 \times 25(\mathrm{~mm})$. The sample was cut into a size of 42 $\mathrm{mm}$ in diameter and $4 \mathrm{~mm}$ in thickness by wire cutting. The sample was solution treated at $530^{\circ} \mathrm{C}$ for $6 \mathrm{~h}$, followed by aging treatment at $265^{\circ} \mathrm{C}$ for $2-48 \mathrm{~h}$.

The COOLPIX-4500 optical microscope was used to observe the metallographic structure, and the JSM 6701F scanning electron microscope with energy dispersive spectrometer was used to analyze the microstructure and elements. The Siemens D5000 was used to analyze the phase constitutions, and the aging kinetics equation concerning 14H-LPSO phase was analyzed by electrical conductivity testing with the Avrami equation.

\section{Results and discussion}

\subsection{Microstructure and phase analysis}

Microstructure of the as-cast Mg95.5Zn1.5Y3 alloy is shown in Fig. 1. It can be seen that the microstructure contains $\alpha-\mathrm{Mg}$ dendrites, the gray bulk phases (marked as A) and the fishbone like eutectic phases (marked as B). The EDS analysis of Points $\mathrm{A}$ and $\mathrm{B}$ are shown in Table 1 . The $\mathrm{Zn} / \mathrm{Y}$ atomic ratio at Point A is close to $1: 1$, which is inferred to be the $\mathrm{Mg} 12 \mathrm{ZnY}$ (LPSO) phase, and the $\mathrm{Zn} / \mathrm{Y}$ atomic ratio is approximately 1.5 at Point B, which can be inferred as Mg3Zn3Y2 (W) phase. So, the alloy is mainly composed of magnesium matrix $(\alpha-\mathrm{Mg})$, Mg12ZnY (LPSO) phase and Mg3Zn3Y2 (W) phase. Figure 2 shows the optical microstructure of $\mathrm{Mg} 95.5 \mathrm{Zn} 1.5 \mathrm{Y} 3$ after aging treatment at $265{ }^{\circ} \mathrm{C}$ for different times. It can be found that the new fine lamellar-shaped phase precipitates from the supersaturated $\alpha-\mathrm{Mg}$ matrix in the initial stage of aging

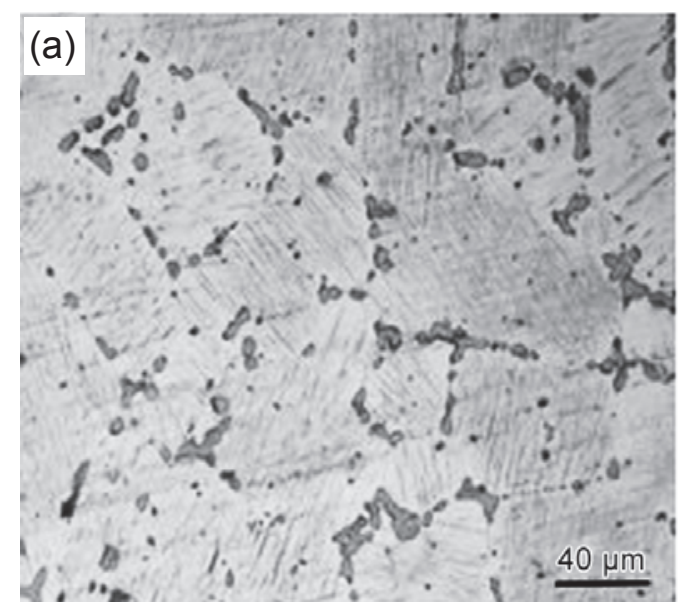

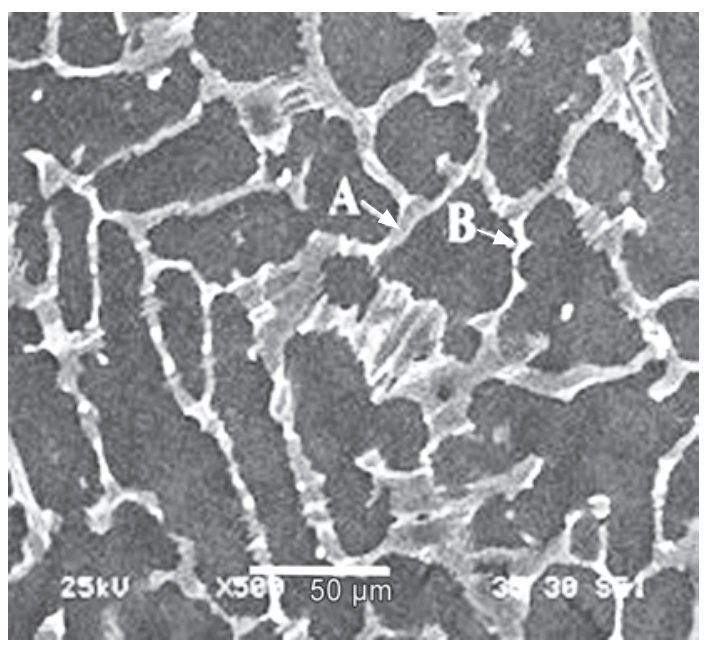

Fig. 1: SEM image of as-cast Mg95.5Zn1.5Y3 alloy

Table 1: EDS analysis of A and B

\begin{tabular}{ccc} 
Point & Element & at.\% \\
\hline & Mg & 93.00 \\
An & Y & 3.76 \\
& Mg & 3.24 \\
\hline \multirow{2}{*}{ B } & Zn & 52.63 \\
& Y & 26.84 \\
& & 20.53
\end{tabular}

treatment, and its volume fraction increases with the increase of aging time. Under a long-term high temperature, the atomic diffusion coefficient and activation energy increase, and the atomic diffusion motion becomes more intense, therefore, the base surface dislocations are continuously formed. The $\mathrm{Zn}$ and $\mathrm{Y}$ atoms continuously fill the faulty surface, resulting in an increase in the volume fraction and size of the precipitated phase. The transmission electron microscopy (TEM) method was used to identify the new lamellar-shaped phase, as shown in Fig. 3. As can be seen from the figure, the distance between the incident spot and the (0002) Mg spot was divided into 14 equal parts, and it can be explained that the fine-grained LPSO phase is the $14 \mathrm{H}-\mathrm{LPSO}$ phase ${ }^{[15-16]}$.

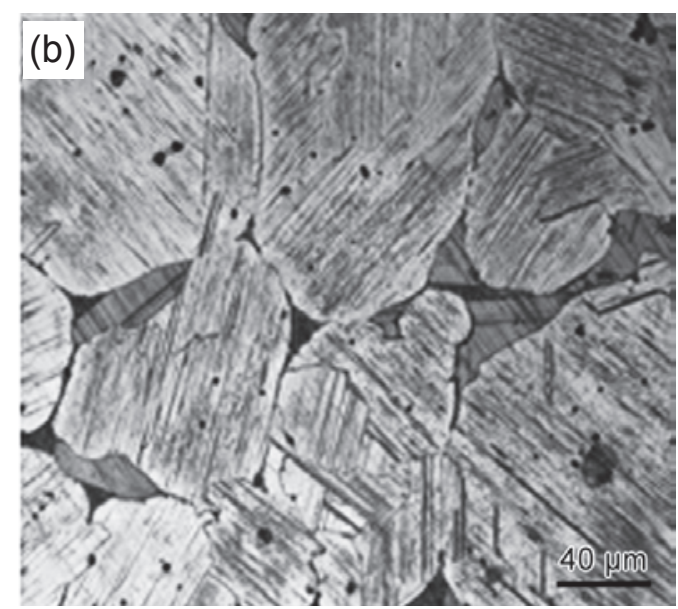



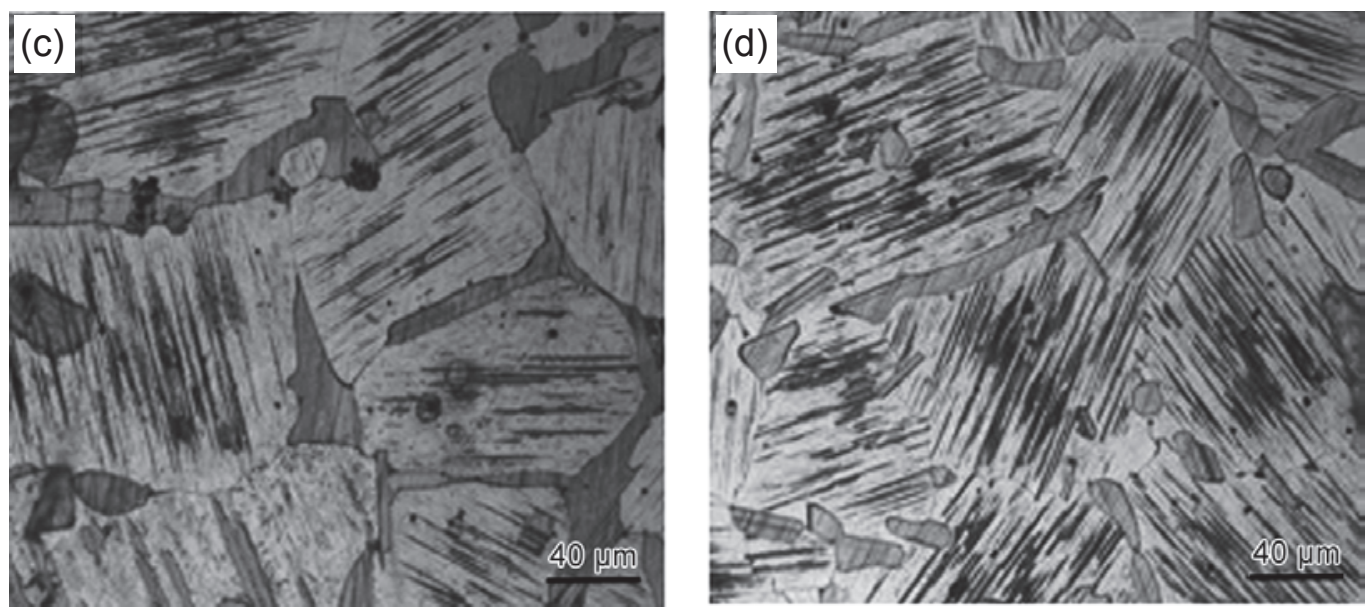

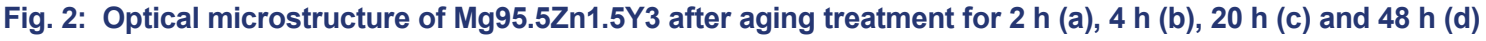
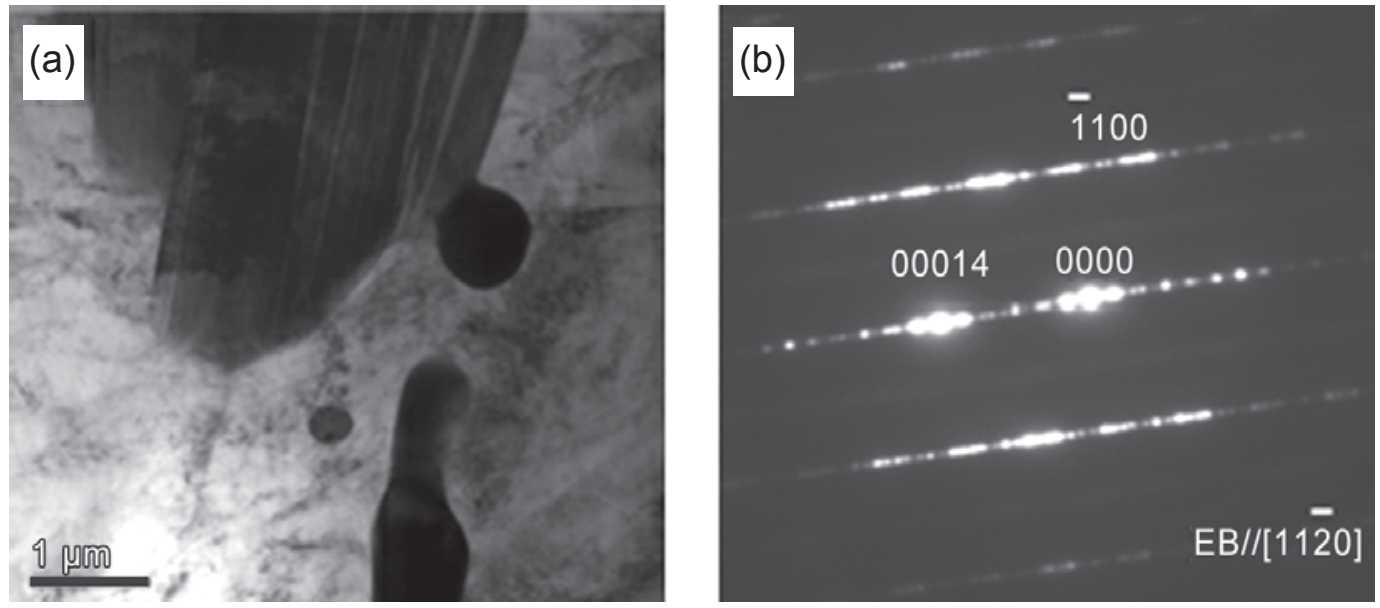

Fig. 3: TEM image (a) and SAED pattern (b) of Mg95.5Zn1.5Y3 after aging treatment at $265^{\circ} \mathrm{C}$ for $2 \mathrm{~h}$

The XRD patterns of Mg95.5Zn1.5Y3 alloys after aging treatment at $265{ }^{\circ} \mathrm{C}$ are shown in Fig. 4. Obviously, the alloys after aging treatment have a new peak of $14 \mathrm{H}$-LPSO phase, and as the aging time increases, the intensity of the diffraction peak of the 14H-LPSO phase increases simultaneously, that is, the content of the $14 \mathrm{H}$-LPSO phase increases, almost covering the whole $\alpha$-Mg grains [Fig. 2(d)]. Besides, it is found that the precipitated $14 \mathrm{H}$-LPSO phase has a random orientation relationship with the secondary phase at grain boundaries and its growth is hindered by grain boundaries. Liu ${ }^{[17]}$ observed that the 14H-LPSO phase firstly precipitated at grain boundaries and then grew into $\alpha-\mathrm{Mg}$ solid solution during the aging treatment of ascast Mg97Zn1Y2 alloy, while 14H-LPSO phase was generated from the supersaturated $\alpha-\mathrm{Mg}$ solid solution by spinodal decomposition in the as-extruded Mg97Zn1Y2 alloy. In our experiment, the precipitation process of as-cast $\mathrm{Mg} 95.5 \mathrm{Zn} 1.5 \mathrm{Y} 3$ alloy is similar to that of the as-extruded Mg97Zn1Y2 alloy. The above analysis demonstrates that the precipitation process of 14H-LPSO phase has no relationship with the state of alloys. The precipitation of $14 \mathrm{H}-\mathrm{LPSO}$ phase from supersaturated $\alpha-\mathrm{Mg}$ solid solution includes two stages: (1) introducing stacking faults (SFs) into magnesium matrix; (2) concentration of solute atoms $(\mathrm{Zn}, \mathrm{Y}){ }^{[18]}$. In previous studies ${ }^{[19]}$, the $14 \mathrm{H}-\mathrm{LPSO}$ phase

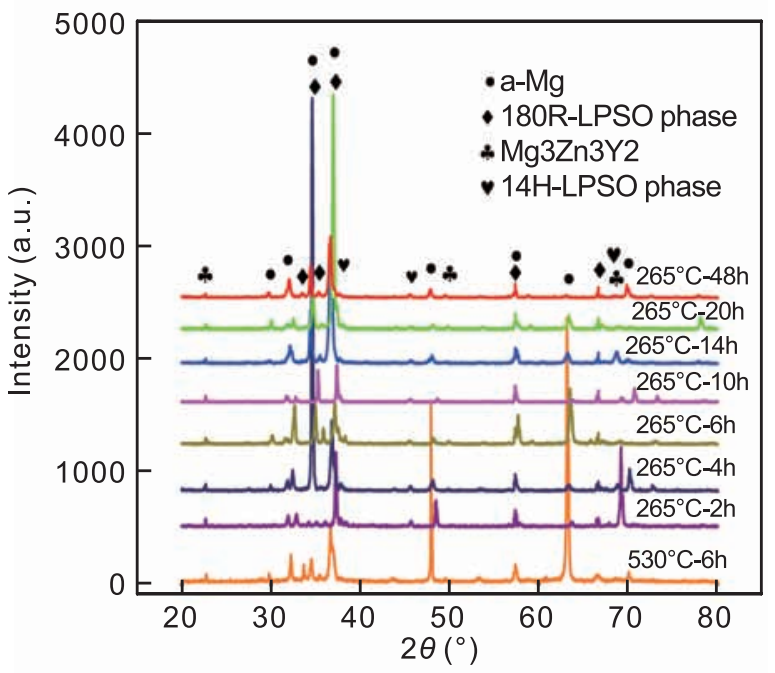

Fig. 4: XRD patterns of Mg95.5Zn1.5Y3 alloy after aging treatment at $265^{\circ} \mathrm{C}$ for different times

mainly precipitated at grain boundaries was ascribed to that SFs were easily generated at grain boundaries due to the large number of defects at grain boundaries. During the solid solution process, most of the secondary phases at the grain boundary are dissolved into the matrix to provide a large amount of $\mathrm{Zn}$ 
and $\mathrm{Y}$ atoms for the formation of the 14H-LPSO phase, which could reduce the stacking fault energy of the matrix and provide favorable conditions for the 14H-LPSO phase precipitated from the supersaturated $\alpha-\mathrm{Mg}$ solid solution.

The 14H-LPSO phase grows continuously in the magnesium matrix and grain boundaries by step growth in this study. Zhu ${ }^{[20]}$ indicated that the growth of $14 \mathrm{H}-\mathrm{LPSO}$ phase was mainly through a ledge growth mechanism.

The LPSO phase not only grows along the base surface, but also grows along the cylinder direction. The length of the precipitated phase in the matrix is much larger than the width, from which it can be inferred that the growth rate of the LPSO phase along the substrate is much greater than the growth rate along the cylinder ${ }^{[15]}$. It was found that each 14H-LPSO phase grew alone firstly and then slowly stacked freely to form a thicker secondary phase ${ }^{[21]}$.

\subsection{Aging kinetics of 14H-LPSO phase}

Figure 5 shows the relationship between aging time and electrical conductivity. It is found that the conductivity of $\mathrm{Mg} 95.5 \mathrm{Zn} 1.5 \mathrm{Y} 3$ alloy rises rapidly, then, tends to be constant when the aging time is up to $20 \mathrm{~h}$. The critical factor affecting the conductivity of the alloy is the scattering of electrons solute in supersaturated solid solution. The solute dissolves into the matrix during solution treatment, which reduces electrical conductivity. In the initial stage of the aging treatment, the formation of the 14H-LPSO phase consumes $\mathrm{Zn}$ and $\mathrm{Y}$ atoms, and the reduction of the elements leads to a decrease in electron scattering and increases the electrical conductivity of the alloy.

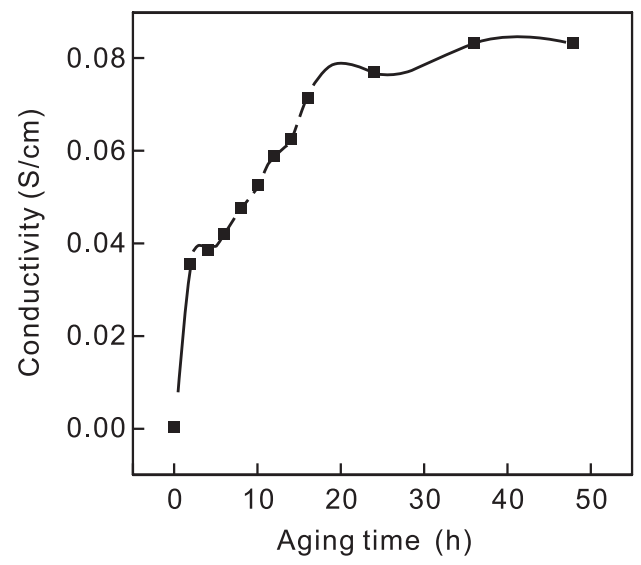

Fig. 5: Effect of aging time on conductivity of Mg95.5Zn1.5Y3 alloy

In the subsequent aging process, the volume fraction and size of lamellar-shaped $14 \mathrm{H}$-LPSO phase increase. The amounts of $\mathrm{Zn}$ and $\mathrm{Y}$ in the matrix decrease rapidly and the precipitation rate of the LPSO phase slows down. When $\mathrm{Zn}$ and $\mathrm{Y}$ atoms in the $\alpha-\mathrm{Mg}$ matrix gradually become insufficient, the conductivity of the alloy tends to be stable. The conductivity is maintained at a relatively high level, up to $0.083 \mathrm{~S} / \mathrm{cm}$.

The Avrami equation can explain the relationship between the precipitation phase and the precipitation time during aging treatment ${ }^{[22]}$ :

$$
f=1-\exp \left[-(b t)^{n}\right]
$$

where $f$ is the volume fraction of precipitated phase, which is the ratio of the amount of precipitated phase at time $t$ to the precipitated phase amount at the end of the precipitation process; $b$ and $\mathrm{n}$ are the rate constant and the Avrami exponent, respectively; where $n$ value depends on the nucleation position and phase transition type. The value of $b$ depends on both the nucleation and growth rates ${ }^{[16]}$. In most cases, it is difficult to obtain the transformed fraction at each time. The conductivity of the alloy is closely related to the precipitation, so conductivity was chosen to represent the volume fraction of the precipitated phase ${ }^{[22]}$ :

$$
f=\frac{K_{t}-K_{0}}{K_{\infty}-K_{0}}
$$

where $K_{t}$ is alloy conductivity at time $t$, and $K_{\infty}$ and $K_{0}$ are the conductivity values at the end and beginning of the transformation, respectively. Taking the logarithm of Eq. (1) gives us:

$$
\lg \left[\ln \left(\frac{1}{1-f}\right)\right]=n \lg b+n \lg t
$$

According to Eq. (3), if the precipitation process of Mg95.5Zn1.5Y3 alloy coincides with the Avrami equation, the plot of $\lg \left[\ln \left(\frac{1}{1-f}\right)\right]$ versus $\lg t$ should exhibit a linear relationship. Meanwhile, $n$ and $\lg b$ should be the slope and the intercept of the $Y$-axis.

Figure 6 shows the curve of $\lg \left[\ln \left(\frac{1}{1-f}\right)\right]$ versus $\lg t$ of the Mg95.5Zn1.5Y3 alloy aging at $265^{\circ} \mathrm{C}$. It can be seen that the $\lg \left[\ln \left(\frac{1}{1-f}\right)\right]$ and $\lg t$ has a linear relationship, which suggests that the precipitation process of the Mg95.5Zn1.5Y3 alloy can be illustrated by the Avrami equation. The $n$ value is 0.6368 in the Avrami equation; as mentioned earlier, $n$ is a constant related to the nucleation position and phase change

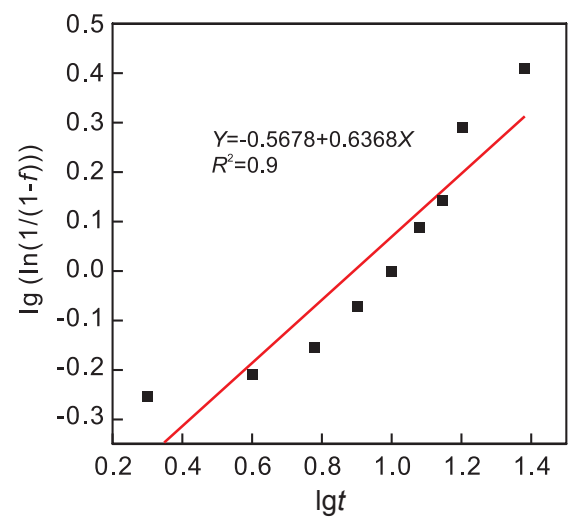

Fig. 6: $\lg (\ln 1 /(1-f)$ vs. $\lg t$ of $M g 95.5 Z n 1.5 Y 3$ alloy aging at $265^{\circ} \mathrm{C}$ 
type, so the phase transition mechanism of Mg95.5Zn1.5Y3 alloy can be explained from the dislocation nucleation ${ }^{[23]}$. Studies ${ }^{[24-25]}$ showed that vacancies, dislocations, stacking faults and grain boundaries and other crystal defects often become the priority part of the non-uniform nucleation of the precipitation phase. On the one hand, these defects can partially offset the lattice distortion caused by the precipitation of phase nucleation. On the other hand, solute atoms accumulate at the defects to form a high concentration area of solute, which easily meets the requirement of solute atom concentration when the precipitate phase formed. According to the literature ${ }^{[26]}$, when the $n$ value is close to 0.5 , the precipitated phase starts to precipitate from the dislocation. Zhu ${ }^{\text {[27] }}$ also reported that 14H-LPSO phase had two building blocks and its growth involved nucleation and lateral gliding of growth ledges. The formation of building blocks includes two steps, that is, a large number of ledges formed firstly, and then, solute atoms diffuse into the two segregation layers. The formation of ledges requires Shockley partial dislocation. Therefore, the obtained results show that the experimental results are consistent with the Avrami equation.

\section{Conclusions}

(1) The microstructure of as-cast Mg95.5Zn1.5Y3 mainly consists of $\alpha-\mathrm{Mg}, \mathrm{W}$ phases and LPSO phases. In the process of aging treatment, fine lamellar-shaped 14H-LPSO phase is formed at the grain boundaries and precipitates from the supersaturated magnesium matrix; and the volume fraction increases as the aging time increases.

(2) Combining electrical conductivity testing with the Avrami equation, the aging kinetics equation about $14 \mathrm{H}-\mathrm{LPSO}$ phase is gained, that is $f=1-\exp \left(-0.2705 t^{0.6368}\right)$. The 14H-LPSO phase precipitated in the matrix is only related to the presence of stacking faults in the matrix and the concentration of $\mathrm{Zn}$ and $\mathrm{Y}$ atoms, and has nothing to do with the presence of solid solution residues at the grain boundaries. The phase transformation mechanism of 14H-LPSO in $\mathrm{Mg} 95.5 \mathrm{Zn} 1.5 \mathrm{Y} 3$ alloy can be described as the change of dislocation energy.

(3) The electrical conductivity of $\mathrm{Mg} 95.5 \mathrm{Zn} 1.5 \mathrm{Y} 3$ alloy increases rapidly with the increase of aging time before $20 \mathrm{~h}$, and then remains almost unchanged as the aging time further increases. With the increase of the aging time, the electrical conductivity tends to be stable. The electrical conductivity remains at a relatively high level, which can reach $0.083 \mathrm{~S} / \mathrm{cm}$.

\section{Acknowledgements}

This work was financially supported by the National Natural Science Foundation of China (51665012), the Jiangxi Province Science Foundation for Outstanding Scholarship (20171BCB23061, 2018ACB21020) and Primary Research \& Development Plan of Jiangxi Province (20192BBEL50019).

\section{References}

[1] Hagiharaa K, Kinoshitab A, Suginob Y, et al. Effect of long-period stacking ordered phase on mechanical properties of Mg97Zn1Y2 extruded alloy. Acta Materialia, 2010, 58: 6282-6293.

[2] Mordike B L. Creep-resistant magnesium alloys. Materials Science and Engineering A, 2002, 324: 103-112.

[3] Tsaia M-H, Chenc M-S, Line L-H, et al. Effect of heat treatment on the microstructures and damping properties of biomedical $\mathrm{Mg}-\mathrm{Zr}$ alloy. Journal of Alloys and Compounds, 2011, 509: 813-819

[4] He S M, Zeng X Q, Peng L M, et al. Microstructure and strengthening mechanism of high strength $\mathrm{Mg}-10 \mathrm{Gd}-2 \mathrm{Y}-0.5 \mathrm{Zr}$ alloy. Journal of Alloys and Compounds, 2007, 427: 316-323.

[5] Hagihara K, Kinoshita A, Sugino Y, et al. Effect of long-period stacking ordered phase on mechanical properties of Mg97Zn1Y2 extruded alloy. Acta Materialia, 2010, 58: 6282-6293.

[6] Oiiorbe E, Garces G, Perez P, et al. Effect of the LPSO volume fraction on the microstructure and mechanical properties of Mg-Y2x-Znx alloys. Journal of Materials Science, 2011, 47(2): 1085-1093.

[7] Qi F G, Zhang D F, Zhang X H, et al. Effects of Mn addition and $X$-phase on the microstructure and mechanical properties of high-strength Mg-Zn-Y-Mn alloys. Materials Science and Engineering A, 2014, 593: 70-78.

[8] Itoi T, Seimiya T, Kawamura Y, et al. Long period stacking structures observed in Mg97Zn1Y2 alloy. Scripta Materialia 2004, 51(2): 107-111.

[9] Xu C, Zheng M Y, Xu S W, et al. Improving strength and ductility of Mg-Gd-Y-Zn-Zr alloy simultaneously via extrusion, hot rolling and ageing. Materials Science and Engineering $A$ 2015, 643: 137-141.

[10] Lee J, Sato K, Konno T J, et al. Stabilization of stacking faults and a long period stacking phase dispersed in $\alpha-M g$ crystalline grains of Mg-0.7at\%Zn-1.4at\%Y Alloy. Materials Transactions, 2009, 50(1): 222-225.

[11] Wu Y J, Peng L M, Zeng X Q, et al. A high-strength extruded Mg-Gd-Zn-Zr alloy with superplasticity. Journal of Materials Research, 2009, 24(12): 3596-3602.

[12] Ding W J, Wu Y J, Peng L M, et al. Formation of 14H-type long period stacking ordered structure in the as-cast and solid solution treated Mg-Gd-Zn-Zr alloys. Journal of Materials Research, 2009, 24(05): 1842-1854.

[13] Wu Y J, Zeng X Q, Lin D L, et al. The microstructure evolution with lamellar $14 \mathrm{H}$-type LPSO structure in an Mg96.5Gd2.5Zn1 alloy during solid solution heat treatment at $773 \mathrm{~K}$. Journal of Alloys and Compounds, 2009, 477(1): 193-197.

[14] Liu H, Xue F, Bai J, et al. Formation behavior of $14 \mathrm{H}$ long period stacking ordered structure in $\mathrm{Mg}-\mathrm{Y}-\mathrm{Zn}$ cast alloys with different $\alpha-M g$ fractions. Journal of Materials Science \& Technology, 2016, 32(12): 1267-1273.

[15] Padezhnova E M, Melnik E V, Milievskii R. Investigation of the Mg-Zn-Y system. Russ. Metall., 1982 (4): 185-188.

[16] Xu C, Zheng M Y, Wu K, et al. Effect of cooling rate on the microstructure evolution and mechanical properties of homogenized Mg-Gd-Y-Zn-Zr alloy. Materials Science and Engineering: A, 2013, 559: 364-370.

[17] Liu H, Bai J, Yan K, et al. Comparative studies on evolution behaviors of $14 \mathrm{H}$ LPSO precipitates in as-cast and as-extruded Mg-Y-Zn alloys during annealing at $773 \mathrm{~K}$. Materials \& Design, 2016, 93: 9-18.

[18] Liu H, Xue F, Bai J, et al. Effect of substitution of 1 at $\% \mathrm{Ni}$ for $\mathrm{Zn}$ on the microstructure and mechanical properties of Mg94Y4Zn2 alloy. Materials Science and Engineering: A, 2013, 585: 387-395. 
[19] Zhang J, Zhang W, Bian L, et al. Study of Mg-Gd-Zn-Zr alloys with long period stacking ordered structures. Materials Science and Engineering: A, 2013, 585: 268-276.

[20] Zhu Y M, Morton A J, Nie J F. The 18R and 14H long-period stacking ordered structures in Mg-Y-Zn alloys. Acta Materialia, 2010, 58(8): 2936-2947.

[21] Adorno A T, Silva R A G, Neves T B. Ag precipitation and dissolution reactions in the $\mathrm{Cu}-3 \mathrm{wt} . \% \mathrm{Al}-4 \mathrm{wt} . \% \mathrm{Ag}$ alloy. Materials Science and Engineering: A, 2006, 441(1): 259-265.

[22] Jackson K A. Kinetic Processes: Crystal Growth, Diffusion, and Phase Transformations in Materials. John Wiley \& Sons, 2006.

[23] Abe E, Kawamura Y, Hayashi K, et al. Long-period ordered structure in a high-strength nanocrystalline Mg-1at.\%Zn2at.\%Y alloy studied by atomic-resolution Z-contrast STEM. Acta Materialia, 2002, 50(15): 3845-3857.
[24] Shogo I, Michiaki Y, Yoshihito K. Relation between corrosion behavior and microstructure of $\mathrm{Mg}-\mathrm{Zn}-\mathrm{Y}$ alloys prepared by rapid solidification at various cooling rates. Corrosion Science, 2009, 51: 395-402.

[25] Peng Q, Guo J, Fu H, et al. Degradation behavior of Mg-based biomaterials containing different long-period stacking ordered phases. Scientific Reports, 2014, 4: 3620-3628.

[26] Xu Z Y. Principle of Phase Change, Science Press, Beijing, 1998.

[27] Zhu Y M, Morton A J, Nie J F. Growth and transformation mechanisms of $18 \mathrm{R}$ and $14 \mathrm{H}$ in $\mathrm{Mg}-\mathrm{Y}-\mathrm{Zn}$ alloys. Acta Materialia, 2012, 60(19): 6562-6572. 